\title{
Bacterial Bloodstream Infections in HIV-infected Adults Attending a Lagos Teaching Hospital
}

\author{
Adeleye I. Adeyemi', Akanmu A. Sulaiman², Bamiro B. Solomon ${ }^{3}$, Obosi A. Chinedu', and Inem A.Victor ${ }^{4}$ \\ 'Department of Microbiology, Faculty of Science, University of Lagos, Akoka, Nigeria, ${ }^{2}$ Department of Haematology and Blood Transfusion, \\ Lagos University Teaching Hospital, Idi-Araba, Nigeria, ${ }^{3}$ Department of Obstetrics and Gynaecology, Bacteriology Research Laboratory, \\ College of Medicine, University of Lagos, Lagos, Nigeria, and ${ }^{4}$ Institute of Child Health and Primary Care, \\ Lagos University Teaching Hospital, Idi-Araba, Nigeria
}

\begin{abstract}
An investigation was carried out during October 2005-September 2006 to determine the prevalence of bloodstream infections in patients attending the outpatient department of the HIV/AIDS clinic at the Lagos University Teaching Hospital in Nigeria. Two hundred and one patients-86 males and 115 females-aged 14-65 years were recruited for the study. Serological diagnosis was carried out on them to confirm their HIV status. Their CD4 counts were done using the micromagnetic bead method. Twenty $\mathrm{mL}$ of venous blood sample collected from each patient was inoculated into a pair of Oxoid Signal blood culture bottles for 2-14 days. Thereafter, $0.1 \mathrm{~mL}$ of the sample was plated in duplicates on MacConkey, blood and chocolate agar media and incubated at $37^{\circ} \mathrm{C}$ for $18-24$ hours. The CD4+ counts were generally low as $67 \%$ of 140 patients sampled had $<200$ cells $/ \mu \mathrm{L}$ of blood. Twenty-six bacterial isolates were obtained from the blood samples and comprised 15 (58\%) coagulase-negative staphylococci as follows: Staphylococcus epidermidis (7), S. cohnii cohnii (1), S. cohnii urealyticum (2), S. chromogenes (1), S. warneri (2), S. scuri (1), and S. xylosus (1). Others were 6 (23\%) Gram-negative non-typhoid Salmonella spp., S. Typhimurium (4), S. Enteritidis (2); Pseudomonas fluorescens (1), Escherichia coli (1), Ochrobactrum anthropi (1), Moraxella sp. (1), and Chryseobacterium meningosepticum. Results of antimicrobial susceptibility tests showed that coagulase-negative staphylococci had good sensitivities to vancomycin and most other antibiotics screened but were resistant mainly to ampicilin and tetracycline. The Gram-negative organisms isolated also showed resistance to ampicillin, tetracycline, chloramphenicol, and septrin. This study demonstrates that co-agulase-negative staphylococci and non-typhoidal Salmonellae are the most common aetiological agents of bacteraemia among HIV-infected adults attending the Lagos University Teaching Hospital, Nigeria. The organisms were resistant to oldergeneration antibiotics often prescribed in this environment but were sensitive to vancomycin, cefotaxime, cefuroxime, and other new-generation antibiotics.
\end{abstract}

Key words: Bacteraemia; Bacterial infections; HIV; HIV infections; Nigeria

\section{INTRODUCTION}

Nigeria has the highest population in Africa, with 1 in 6 Africans being a Nigerian. The rate of HIV/ AIDS prevalence has grown slowly from $1.9 \%$ in 1993 to $5.4 \%$ in 2003, and by the end of 2004, it was approaching $6 \%$ (1). However, a recent survey showed a decline to $4.6 \%$, although the rate var-

Correspondence and reprint requests should be addressed to:

Dr. Adeleye I. Adeyemi

Department of Microbiology

Faculty of Science

University of Lagos

Akoka

Nigeria

Email: adeyemi21@yahoo.com ies from state to state, with some recording as high as $10.6 \%$ (Benue state) and as low as $1.0 \%$ (Ekiti state) (2). The clinical manifestation of HIV secondary infections in developing countries, including Nigeria, shows a high prevalence of infections of the skin, gut, respiratory tract, tuberculosis, and malnutrition $(3,4)$. The virus has a specific capacity to infect the CD4+ class of T lymphocyte, resulting in progressive decline in their (CD4+) number (5). This has serious health consequences since CD4+ cells constitute about $10 \%$ of the total T cell-pool. In AIDS patients, the number of CD4+ cells steadily decreases, and by the time opportunistic infections set in, CD4+ cells may be almost absent. These infections include spirochetal, protozoan, viral, fungal, mycobacterial and pyogenic bacterial infections (6). The later includes gastrointestinal diseases 
caused by enteric bacterial pathogens characterized by high rates of bacteraemia and frequent occurrence of diarrhoea.

Disseminated infections with Salmonella Typhimurium, $S$. Enteritidis, $S$. Arizona, $S$. Dublin, and other non-typhoidal Salmonella serotypes were recognized early in the HIV epidemic (7). Similarly, encapsulated bacteria, including Streptococcus pneumoniae and Haemophilus influenzae are two of the most common bacterial pathogens in HIV-infected persons (8). Pseudomonas aeruginosa has emerged as one of the most common causes of Gram-positive bacteraemia and pneumonia in HIV-infected hospitalized patients, and its incidence in AIDS patients appears to be on the rise, with many studies demonstrating an annual increase in cases (9).

Bacterial bloodstream infections constitute a significant public-health problem and present an important cause of morbidity and mortality in HIVinfected patients. A survey among HIV-I patients in Malawi showed that 30\% had bloodstream infections. Organisms isolated were mainly S. pneumoniae (33\%) and Mycobacterium tuberculosis (28\%) (10). In a retrospective three-year study of all episodes of bloodstream infections in HIV-infected patients in Italy by Bonaldio et al. (11), the most frequent isolates were coagulase-negative staphylococci $(n=33)$, S. aureus $(\mathrm{n}=7)$, Pseudomonas sp. $(\mathrm{n}=7)$, non-typhoid Salmonella $(\mathrm{n}=4)$, and fungi $(\mathrm{n}=1)$. Most patients had a CD4 count of $<100$.

To the best of our knowledge, there has been no previous study on bacterial bloodstream infections of teeming HIV-infected patients that abound in this country, apart from the limited survey carried out by Ogunsola et al. (12). The present study was conducted to determine the status of bacterial bloodstream infections in HIV-infected patients in this environment in relation to their CD4 T lymphocyte counts. This information will assist in identifying clinical predictors of bloodstream infections and in planning appropriate therapy.

\section{MATERIALS AND METHODS}

\section{Study subjects}

Patients were consecutive attendants at the HIV clinic of the Lagos University Teaching Hospital (LUTH). The LUTH is a tertiary-level teaching hospital and referral centre located in Lagos attending HIV-infected patients and others. The study was conducted during October 2005-September 2006.

The cohort consists of HIV-infected individuals referred to or identified at this hospital. The HIVinfected individuals included 86 males and 115 females aged 14-65 years. The entire subjects were attending the outpatient department of the HIV clinic.

The study was approved by the Medical Research and Ethics Committee of the Lagos University Teaching Hospital. Some patients were already on ART before the study commenced.

\section{Serological diagnosis of HIV}

Serum samples obtained from the 201 patients were screened for HIV antibodies by enzyme-linked immunosorbent assay (ELISA) using Murex 1+2 kits (Murex Diagnostic, Dartfold, England). All sera found positive by ELISA were confirmed by Western blot (13).

\section{Determination of CD4}

CD4 was estimated by micromagnetic bead method using kits supplied by Dynal Beads, UK. The procedure involved the addition of micromagnetic bead coated with anti-CD14 antibodies to whole blood. These magnetic beads bind to human monocytes that express anti-CD14 antigens. This is important so that these monocytes which equally express CD4 molecules are removed from the whole blood before quantitation of CD4+ cells. Following the depletion of blood monocytes, the second micromagnetic bead coated with anti-CD4 was added to the monocyte-depleted blood. The blood was placed in the magnetic field so that all the CD4 bearing cells in $100 \mu \mathrm{L}$ of blood were attracted and isolated. These cells were resuspended in $100 \mu \mathrm{L}$ of $5 \%$ glacial acetic acid tinted with Gentian violet and counted under the light microscope using Neubauer counting chamber.

\section{Collection and processing of blood samples}

Venous blood samples $(20 \mathrm{~mL})$ were taken from each patient, and $10 \mathrm{~mL}$ were aseptically inoculated into a pair of Oxoid Signal blood culture bottles system asceptically. The blood samples were subsequently incubated for 2-14 days according to the standards of the World Health Organization (WHO) (14). Thereafter, $0.1 \mathrm{~mL}$ of the sample was drawn using a sterile syringe and plated out on MacConkey, blood and chocolate agar plates using the streak plate technique. Duplicate plates were inoculated for each of the sample. The plates were then incubated at $37^{\circ} \mathrm{C}$ for $18-24$ hours and observed for bacterial growth. The chocolate agar plates were incubated under anaerobic conditions in an anaerobic jar for the possible isolation of microaerophiles.

\section{Characterization and identification of bacterial isolates}

Colonies appearing on the agar plates were subcultured for purity, and a minimum of three colonies 
with identical morphology was selected individually and subjected to identification by standard biochemical tests (15). Finally, the isolates were confirmed using the API 20E and API staph-kits and were later identified using the API 20E (version 4.0), API20 WE (version 6.0), and API STAPH (version 4.0) software. Organisms identified as Staphylococcus were further subjected to coagulase test.

\section{Testing of antimicrobial sensitivity}

Antimicrobial sensitivity test was carried out on each isolate using Oxoid single antibiotics discs, following the recommended standards of the National Committee on Clinical Laboratory Standards (16); 0.5 Macfarland standard of the pure culture of each isolate was inoculated into tryptose soya broth. Using sterile swab sticks, the resulting solution of each isolate was spread over plates of Mueller-Hinton agar until the surface became sticky. Coagulase-negative Staphylococci were screened for susceptibility to the following 17 antibiotics: ceftazidime $(30 \mu \mathrm{g})$, cefotaxime $(30 \mu \mathrm{g})$, ampicillin $(10 \mu \mathrm{g})$, augmentin $(30 \mu \mathrm{g})$, cefuroxime $(30 \mu \mathrm{g})$, tetracycline $(30 \mu \mathrm{g})$, chloramphenicol $(30 \mu \mathrm{g})$, ofloxacillin $(5 \mu \mathrm{g})$, piperacillin-tazobactam (P-100 $\mu \mathrm{g}, \mathrm{TZ}-10 \mu \mathrm{g})$, penicillin (10 unit), oxacillin $(1 \mu \mathrm{g})$, vancomycin $(30 \mu \mathrm{g})$, septrin $(25 \mu \mathrm{g})$, amikacin $(30 \mu \mathrm{g})$, cloxacillin $(5 \mu \mathrm{g})$, erythromycin $(5 \mu \mathrm{g})$, and gentamicin $(10 \mu \mathrm{g})$. The Gram-negative rods were screened for susceptibility to 12 of the listed drugs and nalidixic acid (30 $\mu g)$. The appropriate antibiotic discs were placed on the lawn of bacterial isolates (4 discs per plate). The plates were incubated at $37^{\circ} \mathrm{C}$ for 24 hours after which the zones of inhibition were measured in $\mathrm{mm}$ using a table ruler. The results were recorded as susceptible, intermediate, or resistant. E. coli (NTCC 10148) and S. aureus (ATTCC 12600) were used as control.

\section{RESULTS}

\section{Distribution of age}

The study population comprised $201 \mathrm{HIV}$ patients -86 males and 115 females. They were aged 1465 years with a mean age of 34.3 [standard deviation (SD) 9.9] years (Table 1). Of them, 143 (71.5\%) were aged $20-40$ years. Teenagers and people in the fifth decade seemed to share identical affliction rate (6\%) while those in their fourth decade were more frequently affected (17.5\%). The figure shows the number of study patients when they were first diagnosed HIV-positive.

\section{CD4 range, sex, and antiretroviral status}

Table 2 shows the CD4 count range, antiretroviral therapy, and bloodstream infections of 140 of the 201 patients. Ninety-four (67.1\%) patients had a CD4

\begin{tabular}{|lcccc|}
\hline \multicolumn{5}{|c|}{ Table 1. Age and sex distribution of 201 HIV/ } \\
AIDS patients studied \\
\hline $\begin{array}{l}\text { Age- } \\
\text { group } \\
\text { (years) }\end{array}$ & $\begin{array}{c}\text { Male } \\
(\mathrm{n}=86)\end{array}$ & $\begin{array}{c}\text { Female } \\
(\mathrm{n}=115)\end{array}$ & $\begin{array}{c}\text { Total } \\
(\mathrm{n}=201)\end{array}$ & $\begin{array}{c}\text { Total } \\
\%\end{array}$ \\
\hline $11-20$ & 2 & 10 & 12 & 6.0 \\
$21-30$ & 26 & 44 & 70 & 34.8 \\
$31-40$ & 33 & 40 & 73 & 36.3 \\
$41-50$ & 18 & 15 & 33 & 16.4 \\
$51-60$ & 6 & 6 & 12 & 6.0 \\
$>60$ & 1 & - & 1 & 0.5 \\
\hline
\end{tabular}

count of $<200$ cells $/ \mu \mathrm{L}$ of blood. Seventeen $(65 \%)$ of 26 patients positive for bloodstream infections belonged to this group. The majority (69.6\%) of the patients with CD4 counts above the 200 cells/ $\mu \mathrm{L}$ threshold were on antiretroviral therapy. All the patients but one who recorded $>500$ cells $/ \mu \mathrm{L}$ were female.

\section{Bacterial isolates}

Twenty-six bacterial isolates comprising mainly 15 coagulase-negative staphylococci (58\%) and six non-typhoidal Salmonellae (23\%) were isolated from the blood samples of 26 (12.9\%) of the 201 patients (Table 3). There was an unusual isolation of Ochrobactrum anthropi $(\mathrm{n}=1)$, Moraxella sp. $(\mathrm{n}=1)$, and Chryseobacterium meningospecticum $(\mathrm{n}=1)$ from three patients. Other isolates were $E$. coli $(\mathrm{n}=1)$ and $P$. aeruginosa $(\mathrm{n}=1)$. Altogether, 26 patients-17 females and nine males-had bloodstream bacterial infections.

\section{Antimicrobial susceptibility patterns}

Table 4 shows the antimicrobial susceptibility patterns of the staphylococci isolates to 17 antimicrobial agents while Table 5 shows those of the Gramnegative rods to 13 drugs. The coagulase-negative staphylococci demonstrated good susceptibility patterns to oxacillin and vancomycin. The Gramnegative rods also demonstrated good sensitivi-

Fig. Distribution of HIV-infected patients at first diagnosis at Lagos University Teaching Hospital

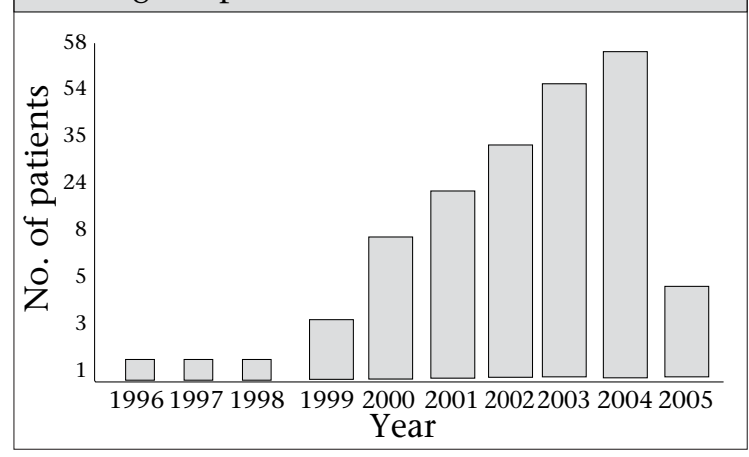




\begin{tabular}{|c|c|c|c|c|c|c|c|}
\hline $\begin{array}{l}\text { CD4 range (cells/ } \\
\text { uL of blood) }\end{array}$ & Male & Female & Total & $\%$ & $\begin{array}{l}\text { No. on } \\
\text { ART (\%) }\end{array}$ & $\begin{array}{c}\text { No. not } \\
\text { on ART (\%) }\end{array}$ & $\begin{array}{l}\text { No. positive } \\
\text { for BSI (\%) }\end{array}$ \\
\hline$<20$ & 9 & 8 & 17 & 12.1 & $0(0)$ & $17(25.0)$ & $6(23.1)$ \\
\hline $21-100$ & 22 & 20 & 42 & 30.0 & 19 (26.3) & $23(33.8)$ & 7 (26.9) \\
\hline $101-200$ & 22 & 13 & 35 & 25.0 & $21(29.2)$ & $14(20.6)$ & $4(15.4)$ \\
\hline $201-500$ & 16 & 19 & 35 & 25.0 & $24(33.3)$ & $11(16.2)$ & $8(30.8)$ \\
\hline $501-1,000$ & 1 & 8 & 9 & 6.4 & $6(8.3)$ & $3(4.4)$ & $1(3.8)$ \\
\hline$>1,000$ & - & 2 & 2 & 1.4 & $2(2.8)$ & $0(0)$ & $0(0)$ \\
\hline Total & 70 & 70 & 140 & 100.0 & $72(100)$ & $68(100)$ & $26(100)$ \\
\hline
\end{tabular}

*61 patients have not done their CD4 count at the time of recruitment into the study; ART=Antiretroviral therapy; BSI=Bloodstream infection; HIV=Human immunodeficiency virus

ty patterns to ceftazidime, cefotaxime, augmentin, cefuroxime, and other antibiotics. Most staphylococci isolates were, however, resistant to ampicillin $(n=11,73.3 \%)$, tetracycline $(n=8,53 \%)$, and penicillin $\mathrm{G}(\mathrm{n}=11,73.3 \%)$. The resistance pattern varied from single (TET or P) to sextiple (AMP-TET-
CM-OFX-P-CN) observed in one S. epidermidis isolate. The Gram-negative rods, on the other hand, were resistant to ampicillin $(\mathrm{n}=7,63 \%)$, tetracycline $(\mathrm{n}=7,63 \%)$, chloramphenicol $(\mathrm{n}=7,63 \%)$, and septrin $(63 \%)$. They showed a pattern varying from single (TET or CAZ) to sextiple (AMP-AMC-TET-

\begin{tabular}{|c|c|c|c|c|}
\hline $\begin{array}{l}\text { Sl. } \\
\text { no. }\end{array}$ & Bacterial isolate & $\begin{array}{l}\text { CD4 count } \\
\text { cells } / \mu \mathrm{L} \\
\text { of blood }\end{array}$ & ART status & $\begin{array}{c}\text { Sex of } \\
\text { patients }\end{array}$ \\
\hline 1 & Staphylococcus scuri & 224 & + & $\mathrm{F}$ \\
\hline 2 & Staphylococcus cohni urealiticum & 221 & + & $\mathrm{F}$ \\
\hline 3 & Staphylococcus epidermidis & 191 & + & M \\
\hline 4 & Salmonella Typhimurium & 55 & - & M \\
\hline 5 & Staphylococcus warneri & 145 & + & $\mathrm{F}$ \\
\hline 6 & Staphylococcus epidermidis & 288 & + & M \\
\hline 7 & Staphylococcus epidermidis & 34 & - & M \\
\hline 8 & Staphylococcus xylosus & 62 & - & $\mathrm{F}$ \\
\hline 9 & Staphylococcus chromogenes & 10 & - & $\mathrm{F}$ \\
\hline 10 & Staphylococcus cohni cohni & 18 & - & $\mathrm{F}$ \\
\hline 11 & Staphylococcus epidermidis & 460 & + & $\mathrm{F}$ \\
\hline 12 & Salmonella Enteritidis & 15 & - & $\mathrm{F}$ \\
\hline 13 & Salmonella Enteritidis & 146 & - & M \\
\hline 14 & Staphylococcus cohni urealiticum & 60 & - & $\mathrm{F}$ \\
\hline 15 & Staphylococcus epidermidis & 6 & - & $\mathrm{F}$ \\
\hline 16 & Staphylococcus epidermidis & 604 & + & $\mathrm{F}$ \\
\hline 17 & Staphylococcus warneri & 46 & - & M \\
\hline 18 & Salmonella Typhimurium & 392 & + & $\mathrm{F}$ \\
\hline 19 & Salmonella Typhimurium & 14 & - & M \\
\hline 20 & Staphylococcus epidermidis & 7 & - & M \\
\hline 21 & Salmonella Typhimurium & 47 & - & M \\
\hline 22 & Escherichia coli & 360 & + & $\mathrm{F}$ \\
\hline 23 & Pseudomonas flourescence & 228 & + & $\mathrm{F}$ \\
\hline 24 & Ochrobactrum anthropi & 220 & + & $\mathrm{F}$ \\
\hline 25 & Moraxella sp. & 110 & - & $\mathrm{F}$ \\
\hline 26 & Chryseobacterium meningospecticum & 88 & - & M \\
\hline
\end{tabular}




\begin{tabular}{|c|c|c|c|c|c|c|c|c|c|c|c|c|c|c|c|c|c|c|}
\hline 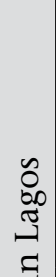 & 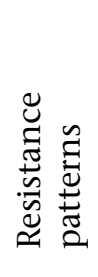 & 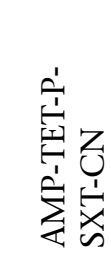 & 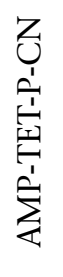 & 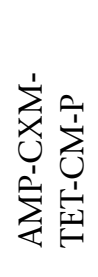 & 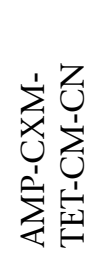 & 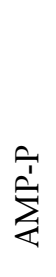 & 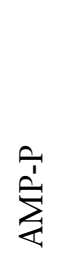 & 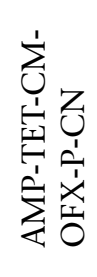 & 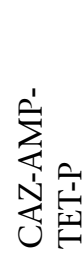 & $\sum_{i}^{\prime}$ & & $a$ & & ' & 产 & 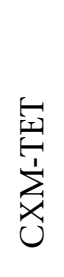 & 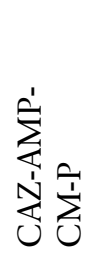 & 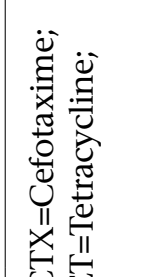 \\
\hline $\overrightarrow{\tilde{c}}$ & Z & $\simeq$ & $\simeq$ & - & $\simeq$ & $n$ & $n$ & $\simeq$ & $\sim$ & $n$ & & $n$ & $n$ & $\omega$ & $n$ & $n$ & $\sim$ & 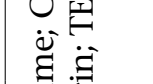 \\
\hline 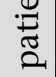 & 工 & s & - & $\infty$ & $\infty$ & $\neg$ & $n$ & $\sim$ & - & $n$ & & $n$ & - & - & $n$ & $\neg$ & $\infty$ & 若 \\
\hline$\stackrel{\Xi}{ \pm}$ & 0 & $n$ & $n$ & $n$ & $n$ & $n$ & $n$ & $n$ & $\sim$ & $n$ & & $n$ & $n$ & 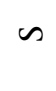 & $n$ & $n$ & $\sim$ & $\underbrace{2}_{\|}$ \\
\hline جُ & 并 & $n$ & $\sim$ & $\omega$ & $\sim$ & $n$ & $n$ & $n$ & $\sim$ & $n$ & & s & $n$ & $\omega$ & $\sim$ & $n$ & $\infty$ & 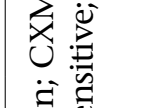 \\
\hline $\begin{array}{l}\Xi \\
\beth\end{array}$ & 离 & $\simeq$ & - & $n$ & - & $n$ & $n$ & $n$ & $n$ & $\sim$ & & 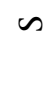 & is & $n$ & $n$ & $n$ & s & 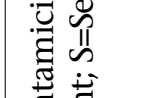 \\
\hline$\underset{\breve{U}}{ت}$ & 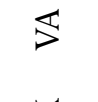 & $n$ & $n$ & $\infty$ & $n$ & $n$ & $n$ & $n$ & $n$ & $\sim$ & & $n$ & is & $\infty$ & $n$ & is & is & 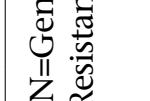 \\
\hline$\Xi$ & ox & $n$ & $n$ & $n$ & $n$ & $n$ & $n$ & $n$ & $\sim$ & $n$ & & $\infty$ & is & $\infty$ & $n$ & is & $\sim$ & 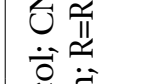 \\
\hline $\begin{array}{l}0 \\
\frac{5}{5} \\
0\end{array}$ & $a$ & $\simeq$ & $\simeq$ & $\simeq$ & $n$ & $\simeq$ & $\simeq$ & $\simeq$ & $\simeq$ & $\simeq$ & & $\simeq$ & $n$ & s & $\simeq$ & is & $\simeq$ & 莺息 \\
\hline $\begin{array}{l}0 \\
0 \\
00 \\
.\end{array}$ & $\stackrel{\mathrm{N}}{\vec{N}}$ & $\sim$ & $\sim$ & $\omega$ & $n$ & $n$ & $n$ & $n$ & $\sim$ & $n$ & & $\infty$ & $n$ & $\omega$ & 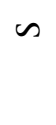 & is & $\sim$ & 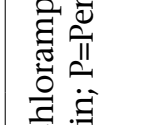 \\
\hline $\begin{array}{l}\tilde{\Xi} \\
\tilde{U} \\
\tilde{u}\end{array}$ & 吕 & $n$ & $n$ & $\omega$ & $\sim$ & $n$ & $n$ & $\simeq$ & $\sim$ & $\sim$ & & $\sim$ & $n$ & $\infty$ & $n$ & $n$ & $n$ & $\sum_{U}^{I I} \overline{\tilde{v}}$ \\
\hline $\begin{array}{l}\ddot{0} \\
.\end{array}$ & $\cup$ & $n$ & $n$ & $\simeq$ & $\simeq$ & $n$ & $n$ & $\simeq$ & $\sim$ & $\sim$ & & $n$ & is & $n$ & $n$ & $n$ & $\simeq$ & \\
\hline 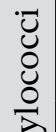 & $\sqrt{t}$ & $\simeq$ & $\simeq$ & $\simeq$ & $\simeq$ & $n$ & $n$ & $\simeq$ & $\simeq$ & $n$ & & $n$ & $\simeq$ & $\omega$ & $n$ & $\simeq$ & $n$ & 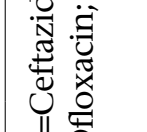 \\
\hline 荵 & 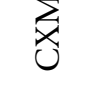 & $\infty$ & $n$ & $\simeq$ & $\simeq$ & $n$ & $n$ & $\sim$ & - & $\sim$ & & $\omega$ & $n$ & $\omega$ & us & $\simeq$ & s & 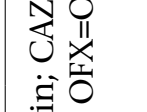 \\
\hline 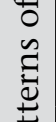 & $\sum_{<}^{U}$ & $\infty$ & $n$ & $\infty$ & $n$ & $n$ & $n$ & $n$ & $\sim$ & $n$ & & $\omega$ & $n$ & $\infty$ & $n$ & $n$ & $n$ & 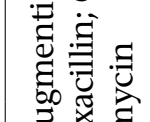 \\
\hline \begin{tabular}{l}
$\tilde{2}$ \\
\multirow{2}{2}{}
\end{tabular} & $\sum$ & $\simeq$ & $\simeq$ & $\simeq$ & $\simeq$ & $\simeq$ & $\simeq$ & $\simeq$ & $\simeq$ & $\simeq$ & & $n$ & $n$ & $\infty$ & $\simeq$ & $n$ & $\simeq$ & 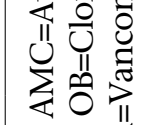 \\
\hline 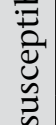 & $\stackrel{凶}{\breve{u}}$ & - & - & $\sim$ & $n$ & $n$ & $n$ & - & - & - & & $n$ & $n$ & $n$ & $n$ & $n$ & $n$ & 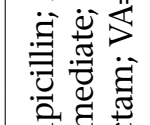 \\
\hline $\begin{array}{l}\frac{\pi}{3} \\
0 \\
0\end{array}$ & 选 & $\sim$ & $n$ & $n$ & $n$ & $n$ & is & $n$ & $\simeq$ & - & & & s & $\infty$ & us & - & $\simeq$ & 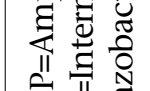 \\
\hline 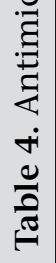 & 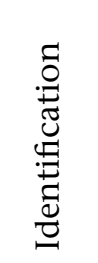 & 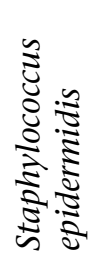 & 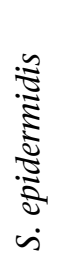 & 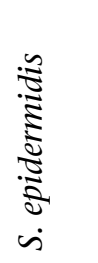 & 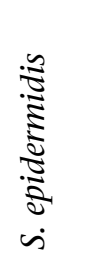 & 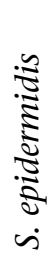 & 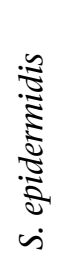 & 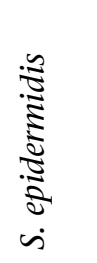 & 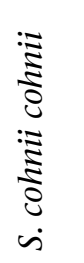 & 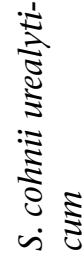 & 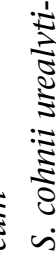 & 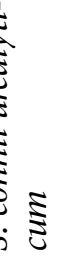 & 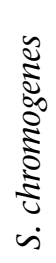 & $\begin{array}{l}\bar{\Xi} \\
\vdots \\
\vdots \\
\dot{s}\end{array}$ & $\begin{array}{l}\bar{\Xi} \\
\vdots \\
\vdots \\
\vdots \\
\dot{s}\end{array}$ & 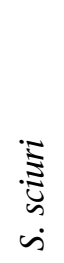 & $\begin{array}{c}\frac{\vec{x}}{2} \\
\frac{0}{\vec{\lambda}} \\
\dot{\omega}\end{array}$ & 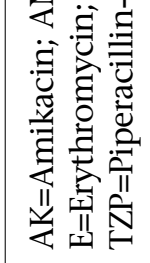 \\
\hline
\end{tabular}




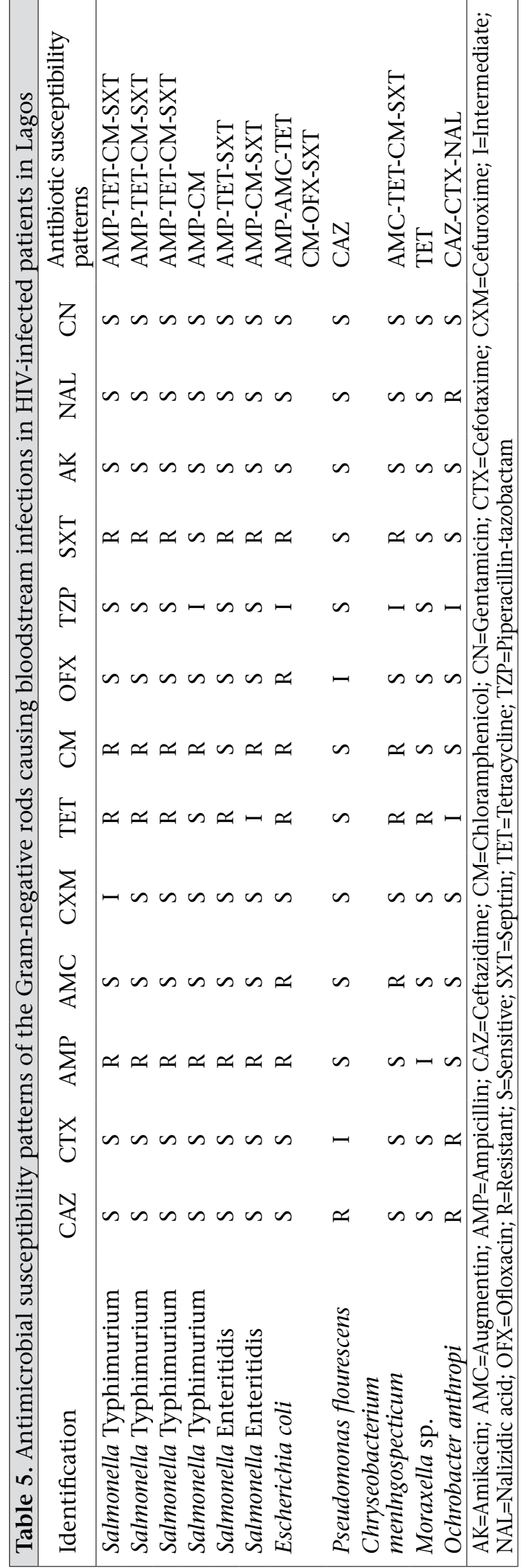

CM-OFX-SXT). The latter was encountered in the E. coli isolate.

\section{DISCUSSION}

The age range of 14-65 years with a mean of 34 years of HIV patients attending the LUTH was observed to be in line with the general picture of HIV/ AIDS infection worldwide, especially in Africa. It has been noted that the pandemic affects mainly sexually-active age-groups $(3,17)$. There were more females than males in the study subjects (115:86), and this is a reflection of disproportionate impact of HIV on women and girls than on men. Obi et al. established that there were more HIV-positive females than males in studies in South Africa, indicating a 'gender bias' (18). The vulnerability of women to HIV in sub-Saharan Africa stem from their greater physiological susceptibility and the severe social, legal and economic disadvantages that confront them (19). Our study also confirmed the upsurge in reported cases of HIV infection in $\mathrm{Ni}$ geria until 2005 when a noticeable decline in infection rate began (2). It was observed in this study that the majority (67\%) of the patients had CD4 counts of $<200$ cells/ $\mu \mathrm{L}$ of blood, and this was more pronounced among subjects who were not yet on antiretroviral therapy. Among the later group were five subjects whose CD4 counts were $<10$ cells/ $\mu \mathrm{L}$. This observation is in agreement with alreadyestablished knowledge of HIV pathogenesis which indicates that there is a steady decline in the CD4 counts of patients and by the time opportunistic infections set in there may be no more CD4 cells present in the immune system (5). The findings of our study also showed that $48.5 \%$ of the subjects had no access to antiretroviral treatment. Some of them had been diagnosed HIV-positive as far back as 2000 and 2001 respectively. This lack of access to medical care, especially the much-needed antiretroviral drugs in Africa, has been widely reported by the WHO and other workers (17). Although this problem is now being addressed in Nigeria at the governmental level, it will take some time before every patient in need will be able to access antiretroviral therapy. Another disturbing fact is that some patients who have been on antiretroviral therapy for a fairly long time had low CD4 counts. This could be attributed to non-adherence to treatment regime and also lack of proper dietary balance. It is known that the many subjects belonged to the low-income group (itenary traders and artisans) and probably could afford the cost of the drugs and nourishable foods which are important ingredients for boosting the population of T cells in their blood. However, the majority of the patients on antiretroviral therapy had their (CD4) counts above the 200 cells $/ \mu \mathrm{L}$ threshold, and nearly all the patients who recorded $>500$ cells $/ \mu \mathrm{L}$ were female. 
Bloodstream infections have long been observed to appear frequently in HIV/AIDS patients. Bekele et al. reported that, of 1,225 hospitalized patients at the University of Florida Health Science Center, 88 (7\%) had bloodstream infections, and 73 of the infections were community-acquired (20). Comparatively, same workers recorded a bacterial infection rate of $12 \%$ in patients with HIV when blood of 201 patients were cultured. Ogunsola et al. reported the infection rate of 33\% in 67 AIDS patients attending the LUTH (12). In the present study, a prevalence of $12.9 \%$ was recorded. In 2000 , Ippolito et al. also recorded $12.1 \%$ cases of bloodstream infections in a prospective multicentre study in HIV patients with advanced stage of the disease (21). Similarly, 30\% prevalence rate of bloodstream infections was recorded in Malawian patients (10).

Bloodstream infections constitute a significant public-health problem and represent an important cause of morbidity and mortality in HIV/AIDS patients. In our study, coagulase-negative Gram staphylococci dominated the number of bacteria isolated as 15 (57.7\%) of the 26 isolates belonged to this group. Gooze and Choi et al. had established that coagulase-negative staphylococci are a major cause of infection in immuno-compromised patients $(22,23)$. Similarly, Bonadio et al. reported that the most frequently-isolated bacteria were coagulase-negative staphylococci (11), and Staphylococcus cohnii had been implicated in community-acquired pneumonia in an HIV-infected patient (24). Other organisms encountered in our study included nontyphoid Salmonellae $(\mathrm{n}=6), P$. fluorescens $(\mathrm{n}=1)$, and E. coli $(\mathrm{n}=1)$. Bonadio et al. also established the presence of non-typhoid Salmonellae, Pseudomonas sp., and one fungus in 68 blood samples from HIVinfected patients with bloodstream infections (11). An increase in the frequency and severity of nontyphoid Salmonella has been reported with AIDS, and infections often present as recurrent diarrhoea with relapsing bacteraemia $(11,12,25)$. $S$. Typhimurium and $S$. Enteritidis are the two most common serotypes isolated from blood of patients with AIDS in the United States (26) and Africa $(7,27)$. This is in agreement with the findings of our study. Invasive non-typhoid Salmonellae are endemic in sub-Sahara Africa, and it has been postulated that transmission between humans-both within and outside health facilities (community-acquired) - may be important (28). P. aerugnosa and other Pseudomonas have also been more frequently recognized as nosocomial bacteraemia in HIV-infected patients (29) and were reported to account for $12 \%$ in hospital patients in Italy (30). Only one of the blood sam- ples cultured grew E. coli. This organism is not frequently isolated in cases of bloodsteam infections among HIV/AIDS patients, and of 861 blood samples examined by Ippolito et al. (21), only seven were positive for $E$. coli. An unusual occurrence was the isolation of $O$. anthropi, C. meningosepticum, and Moraxella sp. Early reports on bloodstream infections had documented the prevalence of encapsulated bacteria and non-typhoid Salmonellae but, during the recent years, broader aetiological spectrum has become evident. The first two cases of $O$. anthropi-associated septicaemia occurring in patient with HIV-infected disease were reported about a decade ago (31). Similarly, although the role of $C$. meningosepticum in immunocompromised host has been recognized (32), clinical data detailing this infection remain limited. Hung et al. reported that the presence of central venous line infection was associated with a poor outcome in patients with $C$. meningosepticum (33). According to Manfredi et al., M. catarrhalis may be responsible for appreciable morbidity among patients with advanced HIV infections with low CD4 counts (34).

Results of antimicrobial susceptibility tests revealed that most coagulase-negative staphylococci were susceptible to most antibiotics screened. Specifically, none was resistant to methicillin and vancomycin. Some of them were resistant to ampicillin, tetracycline, and penicillin. This is in line with results of previous studies. Shittu et al. reported that all 200 isolates of $S$. aureus from clinical samples were susceptible to teicoplanin, vancomycin, fusidic acid, and rifampicin but showed high resistance to penicillin, sulphonamides, and tetracycline (35). Taiwo et al. also reported cumulative resistance of $38-85 \%$ to chloramphenicol, tetracycline, ampicillin, gentamycin, and penicillin $\mathrm{G}$ among bacterial isolates from bloodstream infections in a Nigerian university teaching hospital (36). The isolated Gram-negative bacteria also showed good sensitivity patterns to ceftazidin, cefotaxime, augmentin, and cefuroxime, indicating that they do not produce extended betalactamase. However, they too showed varying resistance to older-generation antibiotics, such as ampicillin, tetracycline, chloramphenicol, and septrin. The multi-drug resistance patterns observed in this study are similar to those earlier reported by Adeleye and Adesoye (37) where septiple resistance comprising SXT-CT-FD-S-C-TETAM was found to dominate in clinical isolates studied. Similarly, Taiwo et al. observed seven patterns of antibiotic resistance, with resistance comprsing the earlier-mentioned antibiotics (AMP-TET-CGM-P) being most common in bloodstream bacterial isolates (36). 
The present study, thus, established that bacterial bloodstream infection is a common occurrence in HIV-positive patients attending the LUTH. The prevalence is comparable with what was obtained in the developed world but less than what is observed in the South African region. The majority of the study patients with bloodstream infection had low CD4 counts, and the antibiotic resistance profile of the bacteria indicate that these are community-acquired. Therefore, a continuous surveillance and intervention strategies should be put in place to manage cases of bloodstream infections in HIV-positive patients in Nigeria.

\section{ACKNOWLEDGEMENTS}

The study was funded by the Central Research Grant, University of Lagos, Akoka, Lagos (Grant No. CRC 2001/19b).

\section{REFERENCES}

1. National Intelligence Council. The next wave of HIV/AIDS: Nigeria, Ethiopia, Russia, India and China. Washington DC: National Intelligence Council, 2002. 32 p.

2. Nigeria. Federal Ministry of Health. National HIV sero-prevalence sentinel survey. Abuja: Federal Ministry of Health, Government of Nigeria, 2009:1-3. (Technical report).

3. Akinsete I, Akanmu AS, Okany CC. Spectrum of clinical disease in HIV-infected adults at the Lagos University Teaching Hospital: a five year experience (19921996). Afr J Med Sci 1998;27:147-51.

4. Akolo CU, Ukoli IJ. Spectrum of clinical diseases at presentation in 200 HIV/AIDS patients at the Jos University Teaching Hospital, Nigeria (abstract). In: Abstracts book of the $14^{\text {th }}$ International Conference on AIDS and STDs in Africa, 2005:TuPODO025.

5. Osmond DH. Classification and staging of HIV disease. In: Cohen PT, Sande MA, Volberding PA, editors. The AIDS knowledge base. $2^{\text {nd }}$ ed. Boston: Little, Brown and Co., 1994:1-13.

6. Sharon S. Woty BC, Sande M. Infections associated with HIV disease. In: Cohen PT, Sande MA, Volberding PA, editors. The AIDS knowledge base. $2^{\text {nd }}$ ed. Boston: Little, Brown and Co., 1994:6.1-6.21.

7. Adeleye IA, Akanmu S, Bamiro S, Smith S, Inem V, Sobande O. Chromosomally mediated antibiotic resistance in non-thyphoidal Salmonellae isolates from HIV patients in Lagos. W Ind Med J 2008;57:548-9.

8. Adeleye A, Uju L, Idika N, Sobande O. Cotrimoxazole resistance in Streptococcus pneumoniae isolated from sputum of HIV-positive patients. W Ind Med J 2008;57:497-9.
9. Hart CA, Beeching NJ, Duerden BI. Infection in AIDS. J Med Microbiol 2000;49:947-67.

10. Archibald LK, McDonald LC, Nwanyanwu O, Kazembe $\mathrm{P}$, Dobbie $\mathrm{H}$, Tokars J et al. A hospital-based prevalence survey of bloodstream infections in febrile patients in Malawi: implications for diagnosis and therapy. J Infect Dis 2000;181:1414-20.

11. Bonadio M, Gigli C, Maccantio O, Longo B, Smorfa A. Bloodstream infection in HIV-positive patients: a review of sixty-eight episodes. J Chemother 1998;10:243-7.

12. Ogunsola FT, Arewa DG, Akinsete IE, Oduyebo OO, Akanmu AS, Odugbemi TO. Aetiology of bacteraemia among adult AIDS patients attending Lagos University Teaching Hospital (LUTH), Lagos, Nigeria. Niger Postgrad Med J 2009;16:186-92.

13. Wilber JC. HIV antibody testing; methodology. In: Cohen PT, Sande MA, Volberding PA, editors. The AIDS knowledge base. $2^{\text {nd }}$ ed. Boston: Little, Brown and Co., 1994:2-7.

14. Miller JM. Specimen collection and processing: blood collection. In: A guide to specimen management in clinical microbiology. $2^{\text {nd }}$ ed. Washington, DC: American Society for Microbiology, 999:53-6.

15. Cowan ST, Steel JK. Manual for the identification of medical bacteria. London: Cambridge University Press, 1965. 238 p.

16. National Committee on Clinical Laboratory Standards. Performance standards for antimicrobial disk susceptibility tests. $8^{\text {th }}$ ed. Approved standard NCCLS Documents M2-M8 and M100-S13(M2).Villanova PA: National Committee on Clinical Laboratory Standards, 2003.

17. Fredriksson J, Kanabus A. HIV and AIDS in Africa. 2005. (www.avert.org/hiv-aids-africa.htm, accessed on 22 February 2007).

18. Obi CL, Onabolu B, Momba MNB, Igumbor JO, Ramalivahna J, Bessong PO et al. The interesting cross-path of HIV/AIDS and water in Southern Africa with special reference to South Africa. Water $S A$ 2006;32:323-43.

19. Joint United Nations Programme on HIV/AIDS. AIDS epidemic update 2009. Geneva: Joint United Nations Programme on HIV/AIDS, 2009:21-2.

20. Afessa B, Morales I, Weaver B. Bacteremia in hospitalized patients with human immunodeficiency virus: a prospective, cohort study. BMC Infect Dis 2001;1:13.

21. Ippolito G, Petrosila N, Viale P, Cristini F, Suter F. Blood stream infections in AIDS patients (abstract). In: Abstracts book of the International Conference on AIDS, Durban, South Africa, 2000:MoPe2292.

22. Gooze L. Bacterial infections associated with HIV. HIV in site knowledge base. California: UCSF Center 
for HIV information, Regents of the University of Califonia (www.hivinsite.ucsf.edu, accessed on 22 February 2007).

23. Choi G, Van den Borne MPJ, Visser CE, Kersten MJ, Kater AP. Invasive infections with a coagulase-negative staphylococcus in an immunocompromised patient: case report and review of the literature. Ann Hematol 2008;87:771-2.

24. Mastroianni A, Coronado O, Nanetti A, Manfredi R, Chiodo F. Community-acquired pneumonia due to Staphylococcus cohnii in an HIV-infected patient: case report and review. Eur J Clin Micro Infect Dis 1995;14: 904-8.

25. Eaton EE, Dobrozycski J, Loas R, Laddis D, Fennelly GJ. Nontyphoidal Salmonella bacteremia and pneumonia as the initial manifestation of human immunodeficiency virus infection in a four-year-old child. AIDS Patient Care STDS 2002;16:247-50.

26. Levine WC, Buehler JW, Bean NH, Tauxe RV. Epidemiology of nontyphoidal Salmonella bacteremia during the human immunodeficiency virus epidemic. $J$ Infect Dis 1991;164:81-7.

27. Kariuki S, Revathi G, Kariuki N, Kiiru J, Mwituria J, Muyodi J et al. Invasive multidrug-resistant nontyphoidal Salmonella infections in Africa: zoonotic or anthroponotic transmission? J Med Microbiol 2006;55:585-91.

28. Morpeth SC, Ramadhani HO, Crump JA. Invasive non-Typhi Salmonella disease in Africa. Clin Infect Dis 2009;49; 606-11.

29. Manfredi R, Nannetti A, Ferri M, Chodo F. Pseudomonas spp complications in patients with HIV disease: an eight-year clinical and microbiological survey. Eur J Epidemiol 2000;16:111-8.
30. Tacconelli E, Tumbarello M, Bertagnolio S, Citton R, Spanu T, Fadda G et al. Multidrug-resistant Pseudomonas aeruginosa bloodstream infections: analysis of trends in prevalence and epidemiology. Emerg Infect Dis 2002;8:220-1.

31. Manfredi R, Nanetti A, Ferri M, Calza L, Tadolini M, Chiodo F. Ochrobactrum anthropi as an agent of nosocomial septicemia in the setting of AIDS. Clin Infect Dis 1999;28:692-4.

32. Chiu C-H, Waddington M, Hsieh W-S, Greenberg D, Schreckenberger PC, Canarhan AM. Atypical Chryseobacterium meningosepticum and meningitis and sepsis in newborns and the immunocompromised, Taiwan. Emerg Infect Dis 2000;6:481-6.

33. Hung PP, Lin YH, Lin CF, Lin MF, Shi ZY. Chryseobacterium meningosepticum infection: antibiotic susceptibility and risk factors for mortality. J Microbiol Immunol Infect 2008;41:137-44.

34. Manfredi R, Nanetti A, Valentini R, Chiodo F. Moraxella catarrhalis pneumonia during HIV disease. $J$ Chemother 2000;12:406-11.

35. Shittu AO, Lin J. Antimicrobial susceptibility patterns and characterization of clinical isolates of Staphylococcus aureus in KwaZulu-Natal province, South Africa. BMC Infect Dis 2006;6:125.

36. Taiwo SS, Fadiora SO, Fayemiwo SA. High antimicrobial resistance among bacterial isolates of blood stream infections (BSI) in a Nigerian University Teaching Hospital. World J Microb Biotech 2007;24:231-6.

37. Adeleye IA, Adetosoye AI. Antimicrobial resistance patterns and plasmid survey of Salmonella and Shigella isolated in Ibadan, Nigeria. East Afr Med J 1993;70:259-62. 